\title{
Softwares educativos aplicados no Ensino de Química: Recursos didáticos potencializadores no processo de aprendizagem
}

\author{
Educational software applied in Chemistry Teaching: Teaching resources that enhance the learning
} process

Software educativo aplicado a la Enseñanza de la Química: recursos didácticos que potencian el proceso de aprendizaje

Recebido: 20/04/2021 | Revisado: 28/04/2021 | Aceito: 04/09/2021 | Publicado: 06/09/2021

\author{
James de Melo Mesquita \\ ORCID: https://orcid.org/0000-0003-1003-4661 \\ Instituto Federal de Educação, Ciência e Tecnologia do Ceará, Brasil \\ E-mail: jjames.mesquita@gmail.com \\ Lidivânia Silva Freitas Mesquita \\ ORCID: https://orcid.org/0000-0003-3552-0391 \\ Secretaria de Educação do Estado do Ceará, Brasil \\ E-mail: lidivaniafreitas@gmail.com \\ Maria Cleide da Silva Barroso \\ ORCID: https://orcid.org/0000-0001-5577-9523 \\ Instituto Federal de Educação, Ciência e Tecnologia do Ceará, Brasil \\ E-mail: ccleideifcemaraca@gmail.com
}

\begin{abstract}
Resumo
$\mathrm{O}$ uso de softwares educativos pode favorecer a construção dos processos de ensino e de aprendizagem acerca do componente curricular Química, muitas dificuldades são impostas pela descontextualização e abstração, esta praticamente onipresente, nos conteúdos. Desse modo, o presente artigo tem por finalidade realizar um levantamento bibliográfico acerca de softwares aplicados ao ensino de Química, como recursos potencializadores do processo de aprendizagem. O estudo foi concentrado na base de dados Google Acadêmico, onde foram explorados trabalhos científicos na temática das TDICs como aliadas no ensino de Química. Foi realizado um filtro nos trabalhos encontrados na seguinte sequência: análise de títulos, palavras-chave e posteriormente leitura dos resumos, a fim de escolher os trabalhos mais relevantes para fins de elaboração desta pesquisa. Foram selecionados trabalhos que abordavam a aplicação de quatro softwares, julgados os mais significativos e de maior impacto nos resultados obtidos. Os softwares PhET Simulações Interativas, LabVirt, ChemSketch e Avogadro oferecem significativo potencial de impacto na construção da aprendizagem, visto que reproduzem ou simulam a realidade, que não é visíel aos olhos, quando sem o auxílio deste tipo de ferramenta. E com a sociedade se tornando cada vez mais dependente de recursos digitais, enfatizase o quão necessário é que a prática pedagógica docente evolua, a fim de dialogar com os estudantes em uma linguagem que lhes é interessante e comum, a linguagem digital. Nesse sentido, a BNCC ressalta que o desenvolvimento desta linguagem propulsiona o pensamento científico de forma crítica e significativa, favorecendo o exercício da autonomia discente.
\end{abstract}

Palavras-chave: Software educativo; Ensino de química; Aprendizagem.

\begin{abstract}
The use of educational software can favor the construction of teaching and learning processes about the Chemistry curricular component, many difficulties are imposed by the decontextualization and abstraction, which is practically omnipresent, in the contents. Thus, this article aims to carry out a bibliographical survey about software applied to the teaching of Chemistry, as resources that enhance the learning process. The study was concentrated in the Google Academic database, where scientific works on the theme of TDICs as allies in the teaching of Chemistry were explored. A filter was performed on the works found in the following sequence: analysis of titles, keywords and later reading of the abstracts, in order to choose the most relevant works for the purposes of preparing this research. Papers that addressed the application of four software were selected, which were considered the most significant and had the greatest impact on the results obtained. PhET Interactive Simulations, LabVirt, ChemSketch and Avogadro softwares offer significant potential impact on the construction of learning, as they reproduce or simulate reality, which is not visible to the eyes, without the aid of this type of tool. And with society becoming more and more dependent on digital resources, it is emphasized how necessary it is for the teaching pedagogical practice to evolve in order to dialogue with students in a language that is interesting and common to them, the digital language. In this sense, the BNCC emphasizes
\end{abstract}


that the development of this language propels scientific thinking in a critical and significant way, favoring the exercise of student autonomy.

Keywords: Educational software; Chemistry teaching; Learning.

\begin{abstract}
Resumen
El uso de software educativo puede favorecer la construcción de procesos de enseñanza y aprendizaje sobre el componente curricular de Química, muchas dificultades son impuestas por la descontextualización y abstracción, prácticamente omnipresente, en los contenidos. Así, este artículo tiene como objetivo realizar un relevamiento bibliográfico sobre el software aplicado a la enseñanza de la Química, como recursos que potencian el proceso de aprendizaje. El estudio se concentró en la base de datos Google Academic, donde se exploraron trabajos científicos sobre el tema de los TDIC como aliados en la enseñanza de la Química. Se realizó un filtro sobre los trabajos encontrados en la siguiente secuencia: análisis de títulos, palabras clave y posterior lectura de los resúmenes, con el fin de elegir los trabajos más relevantes a los efectos de la elaboración de esta investigación. Se seleccionaron los trabajos que abordaron la aplicación de cuatro software, los cuales fueron considerados los más significativos y de mayor impacto en los resultados obtenidos. Los softwares PhET Interactive Simulations, LabVirt, ChemSketch y Avogadro ofrecen un impacto potencial significativo en la construcción del aprendizaje, ya que reproducen o simulan la realidad, que no es visible a los ojos, sin la ayuda de este tipo de herramienta. Y con la sociedad cada vez más dependiente de los recursos digitales, se destaca la necesidad de que la práctica pedagógica docente evolucione, para dialogar con los estudiantes en un lenguaje que les resulta interesante y común, el digital. En este sentido, el BNCC enfatiza que el desarrollo de este lenguaje impulsa el pensamiento científico de manera crítica y significativa, favoreciendo el ejercicio de la autonomía del estudiante.
\end{abstract}

Palabras clave: Software educativo; Enseñanza de la química; Aprendiendo.

\title{
1. Introdução
}

A importância de aprender Química está além da compreensão de conteúdos conceituais ou memorização de fórmulas. Ela é uma ciência que faz parte do cotidiano da humanidade e como bem define Brown et. al (2005), é a Ciência Central. O entendimento dos princípios básicos que regem esta ciência são fundamentais para compreender os materiais e as transformações sofridas por estes, desde as mais simples às mais complexas. Ou seja, a Química é uma ciência que permite uma contextualização social, cultural, ambiental e histórica como descrito na Base Nacional Comum Curricular (BNCC) (Brasil, 2018) e pode ter sua compreensão facilitada através da atribuição de significado ao que é estudado nos espaços formais de ensino.

São várias as dificuldades encontradas no processo de ensino e de aprendizagem de Química, algumas citadas por Delamuta (2017, p. 53) são: abstração, ensino centrado apenas em livros didáticos e a linguagem utilizada nestes, falta de contextualização, linguagem utilizada pelos professores e falta de relação dos conceitos a serem ensinados com os conhecimentos prévios dos estudantes.

Assim, para que o processo de aprendizagem seja eficiente existem muitos fatores envolvidos. Para os fins do desenvolvimento deste trabalho, um ponto válido a destacar, é que os alunos que hoje cursam a Educação Básica são nativos digitais, e que para atingi-los, para chamar sua atenção, é importante e necessário fazer uso de recursos digitais como aliados na construção de uma prática pedagógica docente correlata com a sociedade atual. Esta, que é fluida, está em constante mudança e exige dos educadores postura semelhante na condução do processo de ensino/ aprendizagem.

Como exemplo, têm-se a pandemia da Covid- 19, ainda vivenciada e cheia de desafios. E em virtude desse período de distanciamento social e impossibilidade de atividades presenciais, os educadores precisaram, sem outra alternativa, se reinventar na construção de uma prática pedagógica, que na maioria das vezes utiliza essencialmente TDICs. Muitas atividades desenvolvidas e recursos que têm sido utilizados nesse período, certamente, ficarão como legado do ponto de vista educacional. Pois como ressaltam Nascimento e Rosa (2020), essas tecnologias quando são utilizadas de forma planejada e aliadas a metodologias ativas têm favorecido o desenvolvimento de habilidades necessárias na sociedade.

A BNCC traz em seu texto, duas competências gerais relacionadas ao uso de tecnologias como habilidades importantes para evolução do aprendizado. Na sua essência, o documento ressalta que para o desenvolvimento científico do estudante é importante que ele conheça e utilize diferentes tecnologias. E que ele deve usar tais tecnologias não somente como recursos de 
suporte no processo construção do conhecimento, como também deve se apropriar e aprender a utilizá-las na resolução de problemas individuais ou coletivos, procurando desenvolver autonomia e protagonismo. Assim, percebe-se que o principal documento norteador das aprendizagens essenciais que todos os alunos devem desenvolver ao longo da educação básica incentiva e ressalta a importância da aplicação das TDICs na educação, como uma ferramenta para auxiliar no desenvolvimento de novas estratégias de aprendizagem, capaz de colaborar significativamente para o processo de construção do conhecimento, nas mais diversas áreas.

Existe um leque de possibilidades acerca de tecnologias, gratuitas e facilmente acessíveis, que podem ser utilizadas construção de uma prática pedagógica docente para o Ensino de Química. Como exemplo, existem plataformas que podem ser utilizadas em atividades gamificadas, que, como ressalta Kaap (2012), irão envolver e motivar os estudantes na construção da aprendizagem. Também vale mencionar os simuladores de Realidade Virtual e Aumentada (RVA), que facilitam a compreensão de conceitos relacionados a estruturas químicas não visíveis e que exigem abstração por parte do aluno para seu completo entendimento. Segundo Ferreira e Santos (2020) uso destas tecnologias proporciona maior interatividade entre os alunos e destes com o ambiente tecnológico criando um novo ambiente atrativo e inovador para a aprendizagem.

Contudo, é válido salientar que para garantir um favorecimento do processo de aprendizagem por meio do uso dessas ferramentas digitais, é necessário realizar planejamento direcionado ao alcance dos objetivos pedagógicos pré-definidos. Dionízio et al. (2019), afirma que havendo reflexão, organização e ressignificação da prática pedagógica pelo professor, podese utilizar esses recursos em diferentes modalidades de ensino (presencial ou a distância) e nos mais variados níveis (fundamental, médio ou superior), podendo possuir cunho experimental, teórico e/ou lúdico.

Dessa forma, este trabalho teve por objetivo realizar um levantamento bibliográfico acerca de softwares educativos aplicados no ensino de Química, como recursos potencializadores do processo de aprendizagem, por meio de abordagens mais interativas, lúdicas e atraentes.

\section{Metodologia}

Este estudo seguiu os preceitos de uma pesquisa com abordagem qualitativa e de natureza aplicada, com caráter exploratório. Compreendeu um estudo bibliográfico, concentrado na base de dados Google Acadêmico, onde foram explorados trabalhos científicos na temática das TDICs como aliadas no ensino de Química.

Foram então buscados trabalhos acerca do efeito de potencialização da aprendizagem de Química com uso de TDICs, para então divulgar softwares, aplicativos ou programas que possam ser utilizados nas aulas e sejam de fácil compreensão e aquisição pelos alunos. Foi utilizado o seguinte conjunto de termos de busca: "softwares educativos AND potencializa a aprendizagem AND ensino de química". Diante dos 16.200 trabalhos encontrados e a fim de selecionar os trabalhos mais relevantes para fins de elaboração desta pesquisa, foram utilizados os seguintes recursos como filtro: recorte temporal 20152020, artigos revisados por pares e então prosseguiu-se com análise de títulos, palavras-chave e posteriormente dos resumos. Os trabalhos selecionados, foram então analisados na íntegra.

Para Mól (2017), a abordagem metodológica qualitativa, em pesquisas voltadas ao ensino de Química, é importante porque dispõe a educação como um processo interativo, que acontece na relação entre professores, estudantes e os conceitos científicos específicos da Química. Assim, foi explorado, nos trabalhos que substanciaram este estudo, o impacto das TDICs como ferramenta potencializadora do processo de ensino/aprendizagem, protagonizado por professores e aluno.

De acordo com Godoy (1995), uma característica que define a pesquisa qualitativa é a visão global necessária acerca de um determinado fenômeno, para sua compreensão. Ou seja, um fenômeno pode ser melhor entendido no contexto em que ocorre, e deve, portanto, ser analisado numa perspectiva integrada, considerando o objeto de estudo a partir da perspectiva das pessoas envolvidas, e dos seus pontos de vista. 
Prodanov e Freitas (2013, p.51) esclarecem que a pesquisa de natureza aplicada visa gerar conhecimentos, com finalidades imediatas e aplicação prática, direcionados à solução de problemas específicos. Os autores também ressaltam que a pesquisa de caráter exploratório tem por finalidade proporcionar mais informações acerca da temática a ser investigada, bem como possui um planejamento flexível, possibilitando um estudo complexo, sob diversos ângulos.

Segundo Lakatos e Marconi (2017), a pesquisa bibliográfica

abrange toda a bibliografia já tornada pública em relação ao tema de estudo, desde publicações avulsas, boletins, jornais, revistas, livros, pesquisas, monografias, teses, artigos científicos impressos ou eletrônicos, material cartográfico e até meios de comunicação oral: programas de rádio, gravações, audiovisuais, filmes e programas de televisão. Sua finalidade é colocar o pesquisador em contato direto com tudo o que foi escrito, dito ou filmado sobre determinado assunto, inclusive conferências seguidas de debates que tenham sido transcritas de alguma forma.

Assim, entende-se que a pesquisa bibliográfica de caráter exploratório é feita por meio da análise de materiais já produzidos, visando o desenvolvimento e/ou modificação de ideias e conceitos sobre o assunto a ser investigado.

\section{Softwares Educativos como Recursos Potencializadores no Ensino de Química}

Por meio de emprego e planejamento docente adequados, as tecnologias podem ser envolvidas em uma abordagem significativa dos conteúdos. Para Machado (2016), a química, entre outras ciências investigativas, também conclama para si uso e aplicação de tecnologias específicas para promover a efetivação da aprendizagem científica. Tais ferramentas denotam sua potencialidade, reforçando a ação docente em sala de aula de modo a favorecer colaborativa e substancialmente a aprendizagem significativa dos conteúdos escolares.

Dentre a diversidade de tecnologias disponíveis para emprego no ensino é válido destacar os softwares educativos, que pela definição de Oliveira, Costa e Moreira (2001) são programas construídos com a finalidade de favorecer os processos de ensino/aprendizagem, ou seja, para construir conhecimento relativo a um conteúdo didático. Jucá (2006) classifica os softwares educativos de acordo com suas características e vantagens em tutoriais, exercício ou prática (quizzes), demonstração, simulação, jogo e monitoramento.

É extremamente válido ressaltar que estes recursos estão associados ao aprimoramento do processo de ensino e de aprendizagem, visto que ampliam, em seus usuários, processos mentais superiores como percepção, atenção e memória, agregando vasto conjunto de informações sobre temas específicos a serem desenvolvidos e compartilhados (Machado, 2016).

Delamuta, Assai e Sanchez Júnior (2020) realizaram uma Revisão Sistemática de Literatura (RSL) visando mapear trabalhos relevantes sobre a utilização de TDIC no ensino de química. Uma das questões do estudo apontava para levantamento das tecnologias mencionadas nas publicações, e os autores observaram que a tecnologia mais utilizada nos trabalhos analisados foram os softwares. Essa constatação pode levar ao questionamento do porquê dentre tantas tecnologias disponíveis, estes recursos são os mais estudados e aplicados no ensino de química. A natureza abstrata dessa ciência, peculiaridade onipresente na química, e a falta de relação entre o que é estudado nos livros (nível microscópico) e a realidade (nível macroscópico) pode ser um entrave no processo de aprendizagem. Neste aspecto, Pauletti (2012) afirma que a utilização de softwares computacionais pode potencializar a aprendizagem no componente curricular em questão, visto que podem promover e multiplicar as formas de visualização da química dita abstrata e não observável.

Nessa mesma perspectiva, Passos et al. (2019) realizaram a aplicação de um software de simulação (PhET Simulações Interativas $^{1}$ ) a estudantes do ensino médio acerca do conteúdo Gases. Os autores destacaram que o fato de a ferramenta ser interativa, dinâmica e de fácil manipulação, ela despertou a curiosidade e o interesse dos estudantes, atendendo a expectativa dos

${ }^{1}$ Disponível em https://phet.colorado.edu/pt_BR/simulations/filter?subjects=chemistry\&type=html\&sort=alpha\&view=grid. Acesso em 17 ago. 2021. 
mesmos que acreditavam que os simuladores poderiam contribuir para a construção do conhecimento acerca do conteúdo estudado. Mais uma vez, ressalta-se que o recurso tecnológico fez a ponte entre os níveis macro e o microscópico, facilitando a compreensão dos conceitos relacionados ao que não se vê.

Batista et al. (2016) (ACD/ChemSketch ${ }^{\circledR 2}$ ) e Batista et al. (2018) (Avogadro ${ }^{\circledR 3}$ ) apresentam em seus trabalhos softwares específicos para o desenho de estruturas químicas, que fornecem ainda as propriedades moleculares, sua otimização e visualização 3D, possuindo a capacidade de nomear as moléculas, conforme a IUPAC. Do ponto de vista da Teoria da Aprendizagem Significativa (TAS) de David Ausubel, Andrighetto et al. (2020) realizaram uma pesquisa em que o ACD/ChemSketch ${ }^{\circledR}$ foi empregado como recurso tecnológico para mediar a construção das macromoléculas, onde desenvolveram de modo cooperativo e participativo atividades de investigação sobre aspectos da borracha, buscando uma abordagem contextualizada acerca do conteúdo de polímeros. Os autores relataram que o emprego adequado do recurso tecnológico para mediar a construção das macromoléculas em ambientes de estudo foi de estimável valor. A utilização destas ferramentas tem um grande potencial pedagógico, no ensino de química orgânica, pois tornam o processo de compreensão das estruturas químicas e suas particularidades mais dinâmico, interativo e com significado.

Outro aspecto em que os softwares podem ser utilizados como recursos facilitadores e potencializadores no processo de construção do conhecimento é na experimentação, quando não é possível que ela aconteça em ambiente físico. Para isso, softwares de simulação, com viés experimental, podem ser implementados na prática pedagógica docente. Nesse ponto de vista, Silveira e Vasconcelos (2017) realizaram um estudo de caso, com estudantes do ensino médio, acerca da importância do uso do software educativo LabVirt ${ }^{4}$ no processo de ensino e aprendizagem na disciplina de Química no tópico radioatividade. Segundo os autores, a utilização do referido software possibilitou que os estudantes realizaram experiências virtuais que relacionaram situações do seu dia a dia com implicações do saber científico fazendo com que o processo de ensino e aprendizagem ocorresse de forma dinâmica e interativa. Os autores ainda relataram que o software instigou os estudantes a pesquisarem e a terem vontade de adquirir o conhecimento, bem como despertou a criatividade dos mesmos.

Também é válido ressaltar a importância de softwares desenvolvidos para dispositivos móveis, conhecidos como aplicativos. Pois, segundo a Pesquisa Nacional por Amostra de Domicílio (PNAD) ${ }^{5}$ realizada pelo Instituto Brasileiro de Geografia e Estatística (IBGE), o telefone móvel é o equipamento mais utilizado para acessar a internet. E em virtude da pandemia da Covid-19, o Ensino Remoto Emergencial (ERE) foi implementado e muitos estudantes, certamente, utilizam smartphones para acompanhar as aulas e realizar atividades inerentes ao planejamento docente. E segundo Gresczysczyn, Camargo Filho e Monteiro (2016), a simplicidade e praticidade para utilização, a troca de informação amigável, a facilidade para instalar os aplicativos nos smartphones oferecem múltiplas funcionalidades, e são aspectos que contribuem para a rápida aceitação e disseminação mundial. Rosa e Rohers (2020) realizaram uma pesquisa em que apresentam algumas possibilidades de aplicativos móveis parra o ensino de química, disponíveis na Google Play Store. Os autores identificaram 221 aplicativos, que abordam os mais diversos conteúdos de química, mostrando a grande diversidade disponível.

Os trabalhos apresentados aqui são bastante significativos, pois são várias as dificuldades encontradas no processo de ensino e de aprendizagem dessa área do conhecimento. E em acordo com Machado (2016), o uso de softwares pode ampliar, em seus usuários, processos mentais superiores como percepção, atenção e memória, agregando vasto conjunto de informações sobre temas específicos a serem desenvolvidos e compartilhados também em sala de aula de modo presencial ou virtual.

\footnotetext{
${ }^{2}$ Disponível em https://www.acdlabs.com/resources/freeware/chemsketch. Acesso em 17 ago. 2021.

${ }^{3}$ Disponível em https://avogadro.cc/. Acesso em 17 ago. 2021.

${ }^{4}$ Disponível em http://www.labvirtq.fe.usp.br/indice.asp?time=9:07:40. Acesso em 20 ago. 2021.

${ }^{5}$ Disponível em https://www.ibge.gov.br/estatisticas/sociais/trabalho/9171-pesquisa-nacional-por-amostra-de-domicilios-continuamensal.html?=\&t=downloads. Acesso em 18 ago. 2021.
} 


\section{Softwares Aplicados no Ensino de Química}

A seguir serão apresentados alguns softwares que, de acordo com levantamento bibliográfico realizado, são utilizados no ensino de química e apresentaram resultados positivos nos trabalhos analisados. As ferramentas a seguir tem como semelhança o fato de aproximarem os estudantes da ciência através da contextualização e da relação entre os modos representacionais micro e macroscópico. Pois a Química é uma ciência prática, simples de contextualizar e visual, e para que haja construção efetiva da aprendizagem é necessário que sejam estabelecidas relações entre os conceitos teóricos (nível microscópico) e a aplicação destas teorias (nível macroscópico), assim os estudantes podem potencializar o ensino, visto que podem refletir criticamente sobre os conteúdos a fim de operacionalizar as melhorias necessárias para a promoção da aprendizagem.

\section{PhET Simulações Interativas}

O PhET Simulações Interativas é um recurso pedagógico digital, gratuito, desenvolvido pela Universidade do Colorado, e dispõe de simulações de química, biologia, física, matemática e ciência da terra, que podem ser aplicadas para discussão teórica dos conteúdos e/ou experimentação virtual. Foi construído em acordo com pesquisas realizadas com estudantes e observação do uso dos simuladores em sala de aula. O PhET foi criado com a finalidade de estimular a investigação científica através da interatividade e do apelo visual para modelos científicos, sejam abstratos ou não.

São disponibilizadas 90 simulações, sendo 53 relacionadas a conteúdos de química e classificadas como Química Geral ou Química Quântica, entretanto no tópico de geral é possível identificar simulações pertinentes à Físico- Química. Para todas as simulações disponibilizadas há um Guia do Professor em PDF criado pela equipe do PhET, que tem como finalidade auxiliar o professor a estruturar suas aulas com o uso do recurso.

\section{LabVirt}

O Laboratório Didático Virtual (LabVirt) é uma plataforma que busca promover a produção e o intercâmbio de conhecimentos na construção de uma educação científica mais contextualizada, menos fragmentada e mais significativa. Bem como estimular o pensamento crítico, o uso do método científico, o gosto pela ciência e principalmente a reflexão e compreensão do mundo. Para isso, ele dispõe de simulações feitas pela equipe do LabVirt a partir de roteiros de alunos de ensino médio das escolas da rede pública; links para simulações e sites interessantes encontrados na Internet; exemplos de projetos na seção "projetos educacionais" e respostas de especialistas para questões enviadas através do site.

A plataforma possui mais de 100 simulações dos mais diversos conteúdos, que são abordados de forma bastante contextualizada. Como por exemplo, na simulação "Radioatividade" o conteúdo é apresentado sob a forma de uma hiatória e a metodologia utilizada é baseada da resolução de problemas. Segundo Silveira e Vasconcelos (2017) o referido assunto é introduzido no contexto em que um garoto se machuca ao sofrer um acidente e ao ser socorrido no hospital, aprende sobre radioatividade a partir do funcionamento do equipamento de raios-x. No decorrer da história são realizadas perguntas sobre o tema em que o estudante poderá interagir a fim de responder o problema proposto. As respostas podem ser realizadas no próprio software. As simulações trazidas nele no geral buscam relacionar os conteúdos químicos com o dia a dia do estudante onde ele é conduzido durante a história a participar efetivamente através da leitura e resolução de problemas.

\section{ChemSketch $^{\circledR}$}

$\mathrm{O} A C D / C h e m s k e t c h \circledR$ é software, gratuito, de modelagem molecular voltado para a criação e modificação de estruturas químicas. Destaca-se por ser acessível, de fácil instalação e de grande utilidade prática, nele é possível explorar uma diversidade de aspectos da química, como: 
[...] construção de estruturas moleculares; estudo dos mecanismos de reações; verificação do ângulo entre as ligações com um paralelo às correspondentes energias de ligação; cálculos de propriedades físico-químicas (massa, fórmula centesimal, volume molar, índice de refração, tensão superficial, constante dielétrica, densidade, entre outros cálculos); observações tridimensionais (3D) de isomeria geométrica cis/trans; numeração de cadeia carbônica com paralelo à nomenclatura dos compostos; verificação do banco de dados de diversas macromoléculas e exemplos de instrumentos/vidrarias de laboratório (Andrighetto et al., 2020).

A utilização desta ferramenta, mediada pelo professor com planejamento direcionado e objetivos definidos, tem potencial pedagógico para auxiliar na construção do conhecimento de diversos conteúdos abordados no ensino médio, como exemplo têm-se isomeria geométrica e espacial. Os quais são assuntos que exigem uma visão espacial e abstração por parte dos estudantes.

\section{Avogadro ${ }^{\circledR}$}

O Avogadro é uma ferramenta gratuita de visualização e edição molecular. Possui código aberto, que foi projetado para uso entre plataformas em química computacional, modelagem molecular, bioinformática, ciência de materiais e áreas relacionadas (Batista et al., 2018).

É uma ferramenta intuitiva e de fácil manuseio, construída para estudantes e pesquisadores devido às suas diversas funcionalidades. Dentre seus principais recursos, é válido destacar a possibilidade de visualização tridimensional, sob a forma de uma animação, das moléculas construídas em diversos ângulos. O que colabora para a compreensão, por parte do estudante, de conceitos de ligações químicas, identificação de ângulos de ligação, torção dos ângulos, hibridações, geometria molecular e forças intermoleculares, assuntos que quando trabalhados com estruturas bidimensionais não mostram simulação real destas fórmulas químicas. Outra funcionalidade desta ferramenta é a nomeação de moléculas em acordo com a IUPAC, bem como também fornece a massa molecular, fórmula química e número de átomos que formam a estrutura.

\section{Considerações Finais}

Os trabalhos avaliados e consultados para elaboração desta pesquisa, permitiram concluir que a utilização de recursos digitais amplia e potencializa a visão dos usuários acerca da compreensão do modo representacional microscópico da Química. Visto que softwares de realidade virtual aumentada reproduzem ou simulam a realidade, que não é visível aos olhos, permitindo a interação virtual, sob uma ótica tridimensional, do estudante com fenômenos abstratos e que exigem imaginação, quando sem o auxílio deste tipo de ferramenta.

Percebe-se também que outro potencial destes softwares, que funcionam como uma ponte entre os modos representacionais da Química, está no fato de possibilitar, de uma maneira inovadora, que os professores possam direcionar os estudantes a fazerem e realizarem experimentos, que eles não poderiam fazer no mundo físico/real pelos mais diversos motivos, seja pelo fato de serem experimentos que oferecem risco ou como supracitado, pela impossibilidade de estar nos espaços físicos devido à pandemia da Covid-19.

Os softwares de simulação, PhET Simulações Interativas e LabVirt, apresentados nesta pesquisa, oferecem significativo potencial de impacto na construção da aprendizagem. Pois apresentam algumas características consideradas positivas, como o fato de serem gratuitos, possuírem facilidade de acesso e interação, interface simples e atrativa, além de trazerem os conteúdos de forma contextualizada, atribuindo significado ao que estudado. O ChemSketch ${ }^{\circledR}$ e o Avogadro $^{\circledR}$ apresentam algumas funcionalidades em comum, e são ferramentas indispensáveis para a construção de uma visão tridimensional das estruturas químicas, quando não há a disponibilidade de modelos moleculares físicos, que por sua vez possuem valor de custeio significativo. As ferramentas são gratuitas e, apesar de não se apresentarem língua portuguesa, são de fácil manuseio e intuitivas. Exigindo dos usuários o conhecimento de algumas palavras-chave na língua inglesa, por exemplo, para manuseio das 
ferramentas.

Diante do exposto é possível afirmar que os softwares apresentados neste trabalho são ferramentas que potencializam e facilitam a aprendizagem dos estudantes acerca dos conteúdos que são abordados com a sua utilização. Ressalta-se também que o uso desses softwares, para que seja eficaz, necessita da mediação docente e de planejamento das atividades.

E com a sociedade se tornando cada vez mais dependente de recursos digitais, enfatiza-se o quão necessário é que a prática pedagógica docente também evolua nessa perspectiva, a fim de dialogar com os estudantes em uma linguagem que lhes é interessante e comum, a linguagem digital. Nesse sentido, a BNCC ressalta que o desenvolvimento desta linguagem propulsiona o pensamento científico de forma crítica e significativa, favorecendo o exercício da autonomia discente.

\section{Referências}

Brasil. (2018). Base Nacional Comum Curricular: educação é a base. http://basenacionalcomum.mec.gov.br/

Batista, G. C., Lima, A. R., Crisóstomo, L. C. S., Marinho, M. M., \& Marinho, E. S. (2016). Softwares para o ensino de Química: Chemsketch® um poderoso recurso didático. Redin-Revista Educacional Interdisciplinar, 5(1).

Batista, G. C., Marinho, E. M., Marinho, M. M., \& Marinho, E. S. (2018). Avogadro no ensino de química: um avançado editor molecular de visualização de um grande potencial pedagógico. Redin-Revista Educacional Interdisciplinar, 7(1).

Brown, T. L., LeMay Jr, H. E., Bursten, B. E. \& Burdge, J. R. (2004). Química: a ciência central. Educação Pearson.

Delamuta, B. H. (2017). Roteiro Instrucional para Professores de Ciências: uma proposta para o uso da WebQuest no Ensino de Química. Dissertação (Mestrado) -Universidade estadual do Norte do Paraná. Cornélio Procópio-PR. 189.

Delamuta, B. H., de Souza Assai, N. D., \& Júnior, S. L. S. (2020). O ensino de Química e as TDIC: uma revisão sistemática de literatura e uma proposta de webquest para o ensino de Ligações Químicas. Research, Society and Development, 9(9).

Dionízio, T. P. (2019). O uso de tecnologias da informação e comunicação como ferramenta educacional aliada ao ensino de Química. EaD em Foco, 9(1).

Ferreira, L. C., \& Santos, A. L. (2020). Realidade virtual e aumentada: um relato sobre a experiência da utilização das tecnologias no Ensino de Química. Scientia Naturalis, 2(1).

Godoy, A. S. (1995). Introdução à pesquisa qualitativa e suas possibilidades. Revista de administração de empresas, 35(2), 57-63.

Gresczysczyn, M. C. C., Camargo Filho, P. S., \& Monteiro, E. L. (2016). Aplicativos educacionais para smartphone e sua integração com o ensino de química. Revista de Ensino, Educação e Ciências Humanas, 17(5), 398-403.

Jucá, S. C. S. (2006). A relevância dos softwares educativos na educação profissional. Ciências \& Cognição, 8.

Kaap, K. M. (2012). The gamification of learning and instruction: game-based methods and strategies for training and education. John Wiley \& Sons.

Lakatos, E. M. \& Marconi, M. A. (2017). Fundamentos de metodologia científica. (8a ed.), Atlas.

Lovato, F. L., Michelotti, A., \& Loreto, E. L. S. (2018). Metodologias ativas de aprendizagem: uma breve revisão. Acta Scientiae, 20(2).

Machado, A. S. (2016). Uso de softwares educacionais, objetos de aprendizagem e simulações no ensino de química. Revista Química Nova na Escola, 38(2), 104-111.

Mól, G. S. (2017). Pesquisa qualitativa em ensino de química. Revista Pesquisa Qualitativa, 5(9), 495-513.

Nascimento, F. G. M., \& Rosa, J. V. A. (2020). Princípio da sala de aula invertida: uma ferramenta para o ensino de química em tempos de pandemia. Brazilian Journal of Development, 6(6), 38513-38525.

Oliveira, C. C., Costa, J. W., \& Moreira, M. (2001). Ambientes informatizados de aprendizagem: produção e avaliação de software educativo. Papirus.

Pauletti, F. (2012). Entraves ao ensino de química: apontando meios para potencializar este ensino. Revista Areté| Revista Amazônica de Ensino de Ciências, 5(8), 98-107.

Passos, I. N. G., dos Santos Sousa, J. L., de Sousa, S. F., \& Leal, R. C. (2019). Utilização do software PhET no ensino de química em uma escola pública de Grajaú, Maranhão. Revista Observatório, 5(3), 335-365.

Prodanov, C. C., \& Freitas, E. C. (2013). Metodologia do trabalho científico: métodos e técnicas da pesquisa e do trabalho acadêmico. (2a ed.), Editora Feevale. Rosa, A. S., \& Roehrs, R. (2020). Aplicativos móveis: algumas possibilidades para o ensino de Química. Research, Society and Development, 9(8).

Silveira, F. A., \& Vasconcelos, A. K. P. (2017). Investigação do uso do software educativo LABVIRT no Ensino de Química. Revista Tecnologias na Educação, 23(9), 1-13. 